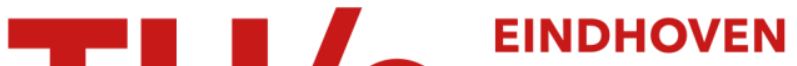

\section{Density functional theory for the elastic moduli of a model polymeric solid}

\section{Citation for published version (APA):}

Sushko, N., Schoot, van der, P. P. A. M., \& Michels, M. A. J. (2003). Density functional theory for the elastic moduli of a model polymeric solid. Journal of Chemical Physics, 118(14), 6594-6604.

https://doi.org/10.1063/1.1558315

DOI:

10.1063/1.1558315

Document status and date:

Published: 01/01/2003

\section{Document Version:}

Publisher's PDF, also known as Version of Record (includes final page, issue and volume numbers)

\section{Please check the document version of this publication:}

- A submitted manuscript is the version of the article upon submission and before peer-review. There can be important differences between the submitted version and the official published version of record. People interested in the research are advised to contact the author for the final version of the publication, or visit the $\mathrm{DOI}$ to the publisher's website.

- The final author version and the galley proof are versions of the publication after peer review.

- The final published version features the final layout of the paper including the volume, issue and page numbers.

Link to publication

\section{General rights}

Copyright and moral rights for the publications made accessible in the public portal are retained by the authors and/or other copyright owners and it is a condition of accessing publications that users recognise and abide by the legal requirements associated with these rights.

- Users may download and print one copy of any publication from the public portal for the purpose of private study or research.

- You may not further distribute the material or use it for any profit-making activity or commercial gain

- You may freely distribute the URL identifying the publication in the public portal.

If the publication is distributed under the terms of Article 25fa of the Dutch Copyright Act, indicated by the "Taverne" license above, please follow below link for the End User Agreement:

www.tue.nl/taverne

Take down policy

If you believe that this document breaches copyright please contact us at:

openaccess@tue.nl

providing details and we will investigate your claim. 


\title{
Density functional theory for the elastic moduli of a model polymeric solid
}

\author{
Nazar Sushko, ${ }^{\text {a) }}$ Paul van der Schoot, and M. A. J. Michels \\ Group Polymer Physics, Department of Applied Physics and Dutch Polymer Institute, \\ Technische Universiteit Eindhoven, P.O. Box 513, 5600 MB Eindhoven, The Netherlands
}

(Received 29 July 2002; accepted 14 January 2003)

\begin{abstract}
We apply a recently developed density functional theory for freely hinged, hard polymeric chains to calculate the elastic moduli of an idealized polymeric solid lacking long-range bond order. We find that for such a model packing effects dominate the elastic behavior of the polymeric solid in a similar way as is the case in the hard-sphere crystal, which we reexamine. Our calculations show that the elastic stiffness of the model polymeric solid is essentially determined by how far one is removed from its melting point. The main role of the chain connectivity is to destabilize the solid relative to the equivalent solid of hard monomers. Comparison of our results with experimental data on semicrystalline polymers shows order-of-magnitude agreement. () 2003 American Institute of Physics. [DOI: 10.1063/1.1558315]
\end{abstract}

\section{INTRODUCTION}

Considering the enormous technological and economic impact solid-state polymers have as construction materials, it is not entirely surprising that a considerable effort is being put into understanding the nature of the elastic behavior of polymeric solids. Scientifically the issue is also of some interest, as the elastic behavior is presumably closely connected with the complex (and often nonequilibrium) structure of the polymeric solid. Focusing our discussion on equilibrium theoretical studies of the elastic moduli of polymers, approaches of various levels of sophistication can be distinguished. The simplest treat the solid as consisting of noninteracting, fully extended chains. More realistic treatments include interactions between neighboring chains within a force-field approximation. The most sophisticated but not necessarily the most accurate calculations rely on quantum-mechanical density functional theory. Before presenting an alternative to these approaches, representing for polymers a novel microscopic description of the elasticity of the solid phase, we first briefly discuss the conventional methods.

Treloar $^{1}$ considered the intrinsic elastic properties of individual, single chains, and found for the stiffness of a model solid of extended polyethylene chains in terms of the Young modulus a value of $182 \mathrm{GPa}$. This is quite close to the experimental values of 100-150 GPa found for highly stretched polyethylene fibers (such as Dyneema ${ }^{\circledR}$ ). ${ }^{2}$ On the other hand, experimental values for so-called isotropic samples of polyethylene are typically less than $2 \mathrm{GPa}$. Clearly, other mechanisms than pure chain stretching need to be involved, at least for unstretched, partially crystallized samples. As is well known, the degree of crystallinity for most solid polymeric materials is below $90 \%$. $^{3}$ In these materials the chains are rarely fully extended, but are packed into folded structures; presumably the entanglements between the polymeric chains present in the melt are in some

${ }^{a)}$ Electronic mail: n.b.sushko@phys.tue.nl way frozen in upon solidification, and form glass-like solid regions throughout the sample.

Tashiro et al. ${ }^{4-6}$ calculated the elastic moduli of chemically realistic polymeric solids beyond the single-chain approach using the force-field technique, in which a test chain is deformed in the presence of the force field from the neighboring chains that interact with this test chain. They assumed the crystal structure to be ideal, with the chains in the crystal fully stretched. The crystal densities obtained from the calculations are within $5 \%$ of the experimental values, and good agreement with experimental values of the Young's modulus of (stretched samples of) polyethylene, aromatic polyamides, poly- $p$-benzamide, and other crystalline polymers were obtained along the chain direction in the crystal, and somewhat less good perpendicular to that (but still of the same order of magnitude).

Finally, there are the quantum-mechanical studies of the elastic moduli of (again perfectly ordered) polymeric solids of, e.g., crystalline polyethylene, and the novel rigid-rod polymer PIPD, based on quantum-mechanical density functional theory. ${ }^{7,8}$ The predictions of these (highly involved, "parameter-free") calculations are quite reasonable when it comes to the Young's modulus along the chain direction, but fail for the bulk modulus with predictions that are several times larger than results known from experiment. ${ }^{7}$ This is indicative of an incorrect description of interchain interactions. We further note that the crystal density used, e.g., in Ref. 8, enters their calculations not self-consistently, but were put in by hand.

Apart from not considering the disordered regions typical of most crystalline polymers, all approaches mentioned so far either seriously underestimate or even completely ignore the role of entropy, although long ago Flory pointed out the importance of entropy in stabilizing a polymer crystal. ${ }^{9}$ It is furthermore well established that for simple liquids entropic packing-effects dominate the microscopic structure of the system, and the same appears to be true for polymers. ${ }^{10}$ Thus, despite that the precise point of crystallization may (in reality) be determined by enthalpic effects, the structure of 
the solid phase is probably still entropy dominated. Note that hard-core models (in which entropy predominates) have been applied reasonably successfully in studies of the crystallization of colloidal particles and model atoms. ${ }^{11}$

Here, we study the generic features of polymer elasticity and the role of packing effects by relying on model polymers with hard-core interactions between the beads. We apply classical liquid-state density functional theory (DFT) to calculate the elastic moduli of idealized polymeric crystals described in more detail in the following. Polymeric fluids have been studied using density functional methods in various contexts before. Perhaps the most relevant to the problem at hand is the work of McCoy and co-workers, ${ }^{10}$ which is an application of polyatomic DFT to chemically realistic polymeric systems. Our approach differs from that of McCoy and co-workers in that information about the connectivity not only enters through the direct correlation function of the polymers in the melt, but also through the contribution of polymeric corrections to the free energy of the crystal.

There are essentially four types of DFT for simple liquids. ${ }^{12}$ The first relies on a thermodynamic perturbation expansion around a liquid state, i.e., the thermodynamic properties of the solid are computed by means of a formal expansion around those of the liquid. This theory was developed by Ramkrishnan and Yussouff (RY), ${ }^{13}$ and is straightforward to implement, i.e., is computationally convenient. The second type of DFT applies a so-called weighted-density approximation (WDA) ${ }^{14}$ in which the solid is treated as an inhomogeneous liquid. The third type of DFT involves an effective-liquid approximation (ELA), ${ }^{15}$ which is a modification of the weighted-density approximation. The difference between WDA and ELA is rather technical and lies in the description of the crystal density in the excess free energy. The fourth type of DFT is the fundamental measure theory (FMT) developed by Rosenfeld, ${ }^{16}$ and later modified by Tarazona, ${ }^{17}$ which relies on functional interpolation between the zero-dimension limit for the excess free energy and properties of the three-dimensional bulk.

The latter three approaches are generally taken to be superior to the former, but are much more involved to implement for fluids of model polymers than for fluids of spherical particles. This in fact is the reason why we developed a polymeric version of the RY DFT in Ref. 18. Despite that WDA (or the more accurate "modified WDA") and ELA give much better prediction for the elastic moduli of hard spheres, we show here that a RY-type theory is not as crude as is often thought. ${ }^{15,19}$ Taking into consideration the highly approximate nature of our model polymers, we argue that for our purposes this method is sufficiently accurate. ${ }^{20}$ The advantage of the RY DFT is that it is easily extended to describe polymeric solids. At this point we contend with a qualitative analysis of the problem.

The method we follow to calculate the elastic moduli from the RY-type DFT is closely connected to the one advanced by Jarić and Mohanty, ${ }^{21}$ and by Jones, ${ }^{22}$ for hard spheres. Unfortunately, the results obtained by these authors for hard spheres are qualitatively incorrect. Not only do they obtain a negative Poisson ratio, which for most materials and in particular for hard-sphere solid is unphysical, their values of the various elastic moduli also differ significantly from ones obtained by means of computer simulations. ${ }^{23}$ Jaric and Mohanty suggested that adding in the excess free energy functional terms higher than second order in the density inhomogeneities ${ }^{24,25}$ could improve these results. ${ }^{26} \mathrm{We}$ show here that these terms are indeed significant, and that a significant improvement over the original theory is possible. In addition we show that restricting the number of reciprocal lattice vectors used in calculations below a certain minimum can drastically affect the final results.

Our results for hard, polymeric chains indicate that in the hypothetical case where the bond order is completely suppressed, the role of chain connectivity is relatively small, and that interactions between the monomeric units dominate the elastic behavior of the solid phase. Connectivity merely changes the density at which the melt-solid phase transition takes place, ${ }^{18}$ and affects the elastic moduli by influencing the stability of the solid phase. For polymer models with stiff bonds that are less extensible or compressible, we expect that lattice frustration effects, which we found in a previous study, ${ }^{18}$ influence the elastic moduli. As we shall see, this turns out to be so.

The remainder of our paper is organized as follows: In Sec. II we first present our model polymeric solid, and briefly discuss the main principles of our polymeric DFT. For more details the reader is referred to Ref. 18. The formal expressions for the elastic moduli from the density functional theory are given in Sec. III. They differ from those previously derived for hard spheres ${ }^{26}$ in that they contain an explicit dependence on the bonded interaction between the monomeric subunits of the polymers. In Sec. IV we describe the calculation technique in detail, and show that the results for the simple case of hard spheres are in good agreement with the computer simulations, ${ }^{23}$ and improve upon the predictions of Jaric and Mohanty. The results for hard-chain crystals are given in Sec. V. We find that the direct influence of the polymeric connectivity on the values of the elastic moduli is minor. The chain connectivity affects the values of the elastic moduli indirectly by changing the freezing density of the polymeric solid. We compare our results with the elastic moduli for real polymeric materials and find a surprisingly good agreement. The conclusions are presented in Sec. VI.

\section{DENSITY FUNCTIONAL THEORY OF A MODEL POLYMERIC SOLID}

The model polymers we consider are freely hinged chains with an adjustable bond stiffness. Let $g\left(\mathbf{r}, \mathbf{r}^{\prime}\right)$ denote the probability that a bond that starts at position $\mathbf{r}^{\prime}$ ends at $\mathbf{r}$. The following bond probability interpolates between the standard Gaussian and Kuhn models: ${ }^{18}$

$$
\begin{aligned}
g\left(\mathbf{r}, \mathbf{r}^{\prime}\right)= & \frac{\sqrt{6}}{8 \pi^{3 / 2} \xi\left|\mathbf{r}-\mathbf{r}^{\prime}\right| l} \\
& \times\left(\exp -\frac{3\left(\left|\mathbf{r}-\mathbf{r}^{\prime}\right|-l\right)^{2}}{2 \xi^{2}}-\exp -\frac{3\left(\left|\mathbf{r}-\mathbf{r}^{\prime}\right|+l\right)^{2}}{2 \xi^{2}}\right),
\end{aligned}
$$




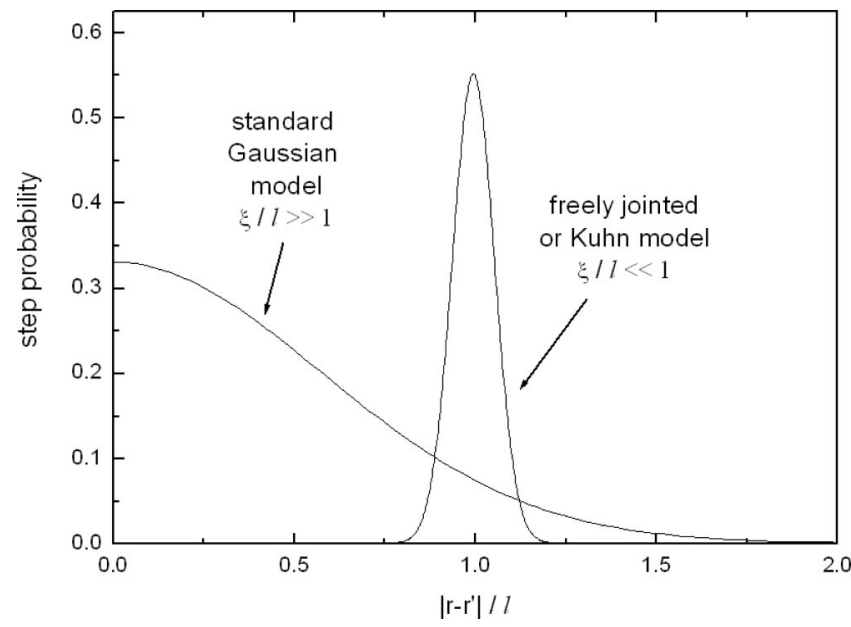

FIG. 1. Step probability for two limiting cases of the polymer model under consideration: a standard Gaussian chain model with $\xi / l \gg 1$ and a freely hinged model with $\xi / l \ll 1$, at fixed Kuhn length $l_{K}$.

where $l$ is a mean length of the bonds, and $\xi$ is a root-meansquare deviation to that. The effective Kuhn length $l_{K}$ of the model depends on $l$ and $\xi$ via the relation $l_{K}=\sqrt{l^{2}+\xi^{2}} \cdot{ }^{18}$ As illustrated in Fig. 1, our model behaves like a freely hinged chain model for $\xi \ll l$ with fixed bond length $l_{K}=l$, whilst for $\xi \gg l$ it becomes equivalent to the Gaussian-chain model with a root-mean-square bond extension $l_{K}=\xi$.

The model polymers are assumed to interact via an isotropic volume exclusion between the beads, which are thought to "live" on a face-centered cubic (fcc) lattice. As argued elsewhere, the only crystal consistent with a freely hinged polymer model consisting of isotropic interaction sites is one where the (phantom) bonds do not display any long-range order. Hence, in our description of the solid the polymers remain random walkers confined to an fcc lattice. (In the reference melt they are random walkers too, but in free, continuous space.) We restrict our calculations of the elastic moduli to the fcc crystal, for it is the only stable structure among the set of all cubic lattices for the aboveintroduced polymer model. ${ }^{18}$
For reasons of computational convenience, the density distribution $\rho(\mathbf{r})$ of material in the crystalline solid phase is often described by a lattice sum of narrow Gaussians. We, too, invoke this approximation, and write for the local segment density ${ }^{21,22}$

$$
\begin{aligned}
\rho(\mathbf{r})= & \frac{\rho_{S}}{N_{c} \pi^{3 / 2} \operatorname{det} \mathbf{M}^{1 / 2}} \\
& \times \sum_{\{\mathbf{R}\}} \exp \left[-(\mathbf{R}-\mathbf{r}) \cdot\left(\mathbf{A} \cdot \mathbf{M} \cdot \mathbf{A}^{\dagger}\right)^{-1} \cdot(\mathbf{R}-\mathbf{r})\right],
\end{aligned}
$$

where $\{\mathbf{R}\}$ indicates the set of all the real-space crystallattice vectors, $\mathbf{M}$ a matrix describing the width of the Gaussian density profiles, $\mathbf{A}$ the lattice-constant matrix with $\mathbf{A}^{\dagger}$ its transpose, $N_{c}$ the number of sites per unit cell, and $\rho_{S}$ the average segment density of the crystal. We do not allow for the presence of the vacancies, so

$$
\rho_{S}=N_{c} \operatorname{det} \mathbf{A}^{-1} .
$$

In the case of cubic symmetry the density profiles are locally isotropic, so $^{21}$

$$
M_{i j}=\alpha \delta_{i j}, \quad A_{i j}=a \delta_{i j}
$$

with $\alpha$ the (dimensionless) square width of the local density profile, measured in units of the lattice constant $a$. Note that for the fcc lattice $N_{c}=4$. In our calculations of the elastic moduli we rely on a description in terms of the reciprocal lattice vectors, which we now define. The reciprocal lattice matrix describing these vectors is given by

$$
\mathbf{B}=2 \pi\left(\mathbf{A}^{\dagger}\right)^{-1},
$$

which for the case of cubic symmetry simplifies to

$$
B_{i j}=b \delta_{i j}
$$

with $b$ the reciprocal-lattice constant for the fcc lattice.

In a previous paper we advanced a density functional theory for the polymeric crystal, ${ }^{18}$ based on an expansion around the liquid or molten state. The grand potential $\Delta \Omega$ of the crystal relative to that of the melt was found to read

$$
\begin{aligned}
\frac{1}{k_{B} T} \frac{\Delta \Omega}{\rho_{L} V} \equiv \Delta \omega= & \frac{1}{\rho_{L} V} \int d \mathbf{r} \rho(\mathbf{r}) \ln \rho(\mathbf{r}) / \rho_{L}-\frac{1}{\rho_{L}^{2} V} \int d \mathbf{r}^{\prime} g\left(\left|\mathbf{r}-\mathbf{r}^{\prime}\right|\right)\left[\rho(\mathbf{r})-\rho_{S}\right]\left[\rho\left(\mathbf{r}^{\prime}\right)-\rho_{S}\right] \\
& -\frac{1}{\rho_{L} V} \sum_{p=2}^{\infty} \frac{1}{p !} \int \ldots \int C^{(p)}\left(\mathbf{r}_{1}, \ldots, \mathbf{r}_{p}\right) \prod_{i=1}^{p} d \mathbf{r}_{i}\left(\rho\left(\mathbf{r}_{i}\right)-\rho_{L}\right) \\
& -\frac{1}{k_{B} T}\left(\mu_{S}-\mu_{L}\right) \frac{1}{\rho_{L} V} \int d \mathbf{r} \rho(\mathbf{r})-\frac{1}{\rho_{L} V} \int d \mathbf{r}\left(\rho(\mathbf{r})-\rho_{L}\right),
\end{aligned}
$$

where for the density distribution $\rho(\mathbf{r})$ we insert the Gaussian profiles Eq. (2). Here, $T$ denotes the temperature, $k_{B}$ Bolzmann's constant, $V$ the volume of the system, $\mu_{S}$ the chemical potential of the solid and $\mu_{L}$ that of the liquid reference phase, and $\rho_{S}$ and $\rho_{L}$ the segment densities of, respectively, the solid and liquid state. $C^{(p)}\left(\mathbf{r}_{1}, \ldots, \mathbf{r}_{p}\right)$ is the $p$-particle direct correlation function of the liquid reference state, which we calculate with the aid of the polymeric 
reference interaction site model (so-called PRISM), ${ }^{27}$ which we derived from an amalgamation of the Lifshitz theory of polymers and the theory of simple liquids. ${ }^{18,28}$ To this end we invoke the usual Percus-Yevick (PY) closure, ${ }^{27}$ mimicking a hard-core interaction between the beads.

The range of the hard-core interactions we denote by $\sigma$, allowing us to define the so-called fusion parameter $\Gamma$ $\equiv l_{K} / \sigma$, which measures the strength of the coupling between the chain connectivity and the effective interactions between the beads. Note that chain connectivity enters our description through the second term of Eq. (7), as well as via the direct correlation functions in the third term. ${ }^{29}$ The second term is accurate only at length scales small compared to the size of the chains. This is one of the reasons why the long-range bond order cannot be established within our model description. (See also the discussion in Ref. 18.)

It is convenient to rewrite Eq. (7) in terms of a Fourier representation of the density distribution Eq. (2),

$$
\rho(\mathbf{r})=\rho_{L}\left[1+\eta+\sum_{\{\mathbf{q}\}} \zeta(\mathbf{q}) \exp i \mathbf{q} \cdot \mathbf{r}\right]
$$

with $\{\mathbf{q}\}$ the set of the reciprocal lattice vectors of the fcc lattice, $\eta=\left(\rho_{S}-\rho_{L}\right) / \rho_{L}$ the dimensionless density jump across the crystallization transition, and

$$
\zeta(\mathbf{q})=(1+\eta) \exp -\frac{1}{4} \mathbf{q} \cdot \mathbf{A} \cdot \mathbf{M} \cdot \mathbf{A}^{\dagger} \cdot \mathbf{q} .
$$

Inserting Eq. (8) into Eq. (7), and truncating the sum containing the direct correlation functions after the third term, we get an expression for the grand potential,

$$
\begin{aligned}
\Delta \omega= & 1-(1+\eta)\left(5 / 2+\ln \frac{N_{c} \pi^{3 / 2} \alpha^{3 / 2}}{(1+\eta)}-\frac{1}{k_{B} T}\left(\mu_{S}-\mu_{L}\right)\right) \\
& -\sum_{\{\mathbf{q}\}} \hat{g}(\mathbf{q}) \zeta^{2}(\mathbf{q})-\frac{1}{2} \eta^{2} \rho_{L} \hat{C}_{L}^{(2)}(0) \\
& -\frac{1}{2} \rho_{L} \sum_{\{\mathbf{q}\}} \zeta^{2}(\mathbf{q}) \hat{C}_{L}^{(2)}(|\mathbf{q}|)-\frac{1}{6} \eta^{3} \rho_{L}^{2} \hat{C}_{L}^{(3)}(0,0),
\end{aligned}
$$

which we minimize with respect to the liquid density $\rho_{L}$, the parameter $\eta$, and also the width of the density profile $\alpha .{ }^{30} \mathrm{In}$ our density functional we include only the zero- $q$ part of the three-body direct correlation function ${ }^{24}$

$$
\hat{C}_{L}^{(3)}(q, 0)=\left.\frac{\partial \hat{C}_{L}^{(2)}(q)}{\partial \rho}\right|_{\rho=\rho_{L}},
$$

since only this term is believed to contribute significantly to the elastic moduli. ${ }^{26}$ Higher order terms are usually deemed to be unimportant, although not much is known about whether this is true or not. ${ }^{24,25}$

The minimization of Eq. (10) was done by a standard quasi-Newton algorithm from the $\mathrm{NAG}^{\circledR}$ library (Mark 18, E04JYF). The number of reciprocal lattice vectors $\{\mathbf{q}\}$ needed to accurately describe the crystal phase was determined empirically (see Sec. IV). The conditions for coexistence between liquid and solid phases are found by equating the pressures and chemical potentials in both phases. In other words, we set $\Delta \omega=-\Delta P / k_{B} T \rho_{L}=0$ and $\mu_{S}-\mu_{L}=0$ in Eq. (7) to ensure phase coexistence, with $\Delta P$ the pressure difference in both phases.

In Sec. III we describe how the elastic moduli can be calculated from the density functional Eq. (10).

\section{ELASTIC MODULI FROM DENSITY FUNCTIONAL THEORY}

To calculate the elastic moduli of our polymer crystal, we use the method proposed for hard-sphere crystals by Jaric and Mohanty, ${ }^{21}$ and adapt it to our needs. We use the usual Einstein convention for the summation of the tensorial components, so Hooke's law may be written as

$$
d \sigma_{i j}=C_{i j k l} d \epsilon_{k l}
$$

with $\sigma_{i j}$ the components of the stress tensor, $\epsilon_{i j}$ the components of the strain tensor, and $C_{i j k l}$ the components of isothermal elastic modulus tensor $(i, j=1,2,3)$. The stress tensor is the first derivative of the Helmholtz free energy $F$ of a deformed crystal with respect to the strain $\epsilon_{i j}$ in the limit of zero strain, $\sigma_{i j}=V^{-1} \partial F /\left.\partial \epsilon_{i j}\right|_{\epsilon=0}$. Jarić and Mohanty ${ }^{21}$ showed that

$C_{i j k l}=\left.\frac{1}{V} \frac{\partial^{2} F}{\partial \epsilon_{i j} \partial \epsilon_{k l}}\right|_{\epsilon=0}+P\left[\delta_{i j} \delta_{k l}-\frac{1}{2}\left(\delta_{i k} \delta_{j l}+\delta_{j k} \delta_{i l}\right)\right]$

with again $P$ the external pressure and $V$ the volume of the system. Equation (13) may be obtained in an isothermal isobaric ensemble by formally expanding the elastic Gibbs free energy $G_{\text {el }}$ for small strain, defined by ${ }^{21}$

$$
G_{\mathrm{el}}=\left(F_{\epsilon}-F\right)+P\left(V_{\epsilon}-V\right)=\frac{V}{2} \epsilon_{i j} C_{i j k l} \epsilon_{k l}+\cdots,
$$

where we drop all terms of higher than second order in the strain, $C_{i j k l}$ is the second derivative of the elastic Gibbs free energy with respect to the components of strain tensor, $F_{\epsilon}$ is the Helmholtz free energy, and $V_{\epsilon}$ the volume, conditions under deformation as indicated by the subscript $\epsilon$.

Since our DFT is most conveniently expressed in the grand canonical ensemble, we seek to represent the elastic Gibbs free energy $G_{\mathrm{el}}$ in term of the grand potential $\Delta \Omega$, which is defined relative to a liquid reference phase. Note that in the thermodynamic limit $\Omega_{\epsilon}=F_{\epsilon}-\mu_{\varepsilon} n$ and $\Omega=F$ $-\mu n$, and that the number of segments $n$ in both the strained and unstrained crystals remains invariant under the deformation. Because we assume strain $\epsilon$ to be small, we expand to second order in $\epsilon$ the volume $V_{\epsilon}$, the chemical potential $\mu_{\epsilon}$, and the Helmholtz free energy $F_{\epsilon}$. We identify the work done on the liquid reference phase against the deformation under the pressure $P_{L}$ as $\Omega_{\epsilon, L}-\Omega_{L}=-P_{L}\left(V_{\epsilon}-V\right)$. Adding and substracting the term $P_{L}\left(V_{\epsilon}-V\right)$ to Eq. (14), and taking into account the expansions of $V_{\epsilon}, \mu_{\epsilon}$, and $F_{\epsilon}$ in $\epsilon$, we finally get ${ }^{21}$

$$
G_{\mathrm{el}}=\Delta \Omega_{\epsilon}+V_{\epsilon}\left(P-P_{L}\right) .
$$

For the unstrained system the grand potential $\Delta \Omega$ $=k_{B} T \rho_{L} V \Delta \omega$ is given by Eq. (10). To obtain the free energy of the strained solid, we follow Jaric and Mohanty and assume the microscopic strain $\zeta$ and the macroscopic strain $\boldsymbol{\epsilon}$ 
to be equal, $\boldsymbol{\zeta}=\boldsymbol{\epsilon}$ (see the arguments and discussion in Ref. 21). These strains are generally thought to be different, and in fact to depend on the type of deformation. ${ }^{31}$ At this point we ignore the issue, and discuss the effect of the nonequality of the microscopic and macroscopic strains in Sec. IV. The strained Gaussian width tensor $\mathbf{M}_{\epsilon}$ of the density profiles differs from the unstrained tensor $\mathbf{M}$ by an amount $\mathbf{\Delta M}$ $=\mathbf{M}_{\boldsymbol{\epsilon}}-\mathbf{M}$, because of the nonaffine character of deformation below the unit-cell (Cauchy) scale. ${ }^{21,22}$ For the case of cubic symmetry we have $M_{\epsilon, i j}=\alpha_{\epsilon} \delta_{i j}$ and $\Delta M_{i j}=\Delta \alpha \delta_{i j}$ with $\Delta \alpha=\alpha_{\epsilon}-\alpha$.

We expand the expression for the elastic free energy around the unstrained state in terms of the strains $\epsilon, \zeta, \Delta \mathbf{M}$. Obviously, the zero- and first-order terms vanish (see again Jaric and Mohanty ${ }^{21}$ for more details), hence only secondorder terms are important. We compute the elastic moduli from these quadratic terms using the results of the minimization of Eq. (7) for the unstrained case. We allow for the relaxation of the density profiles under the strain, ${ }^{22}$ implying that we need to minimize the functional Eq. (7) twice. The first minimization is with respect to the density of the reference liquid $\rho_{L}$, the relative density of the crystal $\eta$, and the equilibrium density profile width $\alpha$. The second minimization is with respect to the variational parameter $\Delta \alpha$.

We thus arrive at the following variational form for the elastic energy $G_{\mathrm{el}}$ :

$$
\begin{aligned}
& G_{\mathrm{el}}=\frac{V}{2} \epsilon: \mathbf{C}: \epsilon=\frac{V}{2} \epsilon_{i j} C_{i j k l} \epsilon_{k l} \\
& =\min _{\Delta \mathbf{M}} \frac{V}{2}\left[\epsilon: \mathbf{C}^{\epsilon \epsilon}: \epsilon\right. \\
& \left.+\mathbf{2 \Delta M}: \mathbf{C}^{\alpha \epsilon}: \epsilon+\Delta M: \mathbf{C}^{\alpha \alpha}: \Delta M\right],
\end{aligned}
$$

where $\min _{\Delta \mathbf{M}}$ denotes a minimization with respect to the components of the tensor $\Delta \mathbf{M}$. We used following definitions for the tensors:

$$
\begin{aligned}
& \mathbf{C}^{\epsilon \epsilon}=C^{\epsilon \epsilon}+C^{\epsilon \zeta}+C^{\zeta \epsilon}+C^{\zeta \zeta}, \\
& \mathbf{C}^{\epsilon \alpha}=C^{\alpha \epsilon}+C^{\alpha \zeta}, \\
& \mathbf{C}^{\alpha \alpha}=C^{\alpha \alpha} .
\end{aligned}
$$

The tensorial blocks of the elastic modulus tensor can be expressed in terms of the components $B_{i j}$ of the reciprocal lattice matrix $\mathbf{B}$ defined in Eq. (5) $\mathrm{as}^{21}$

$$
\begin{aligned}
C_{i j k l}^{\epsilon \epsilon}= & \delta_{i j} \delta_{k l} k_{B} T \rho_{L}(\eta+1)^{2} \frac{\partial^{2} \Delta \omega}{\partial \eta^{2}}, \\
C_{i j k l}^{\epsilon \zeta}=C_{i j k l}^{\zeta \epsilon}= & \frac{1}{2} \delta_{i j} k_{B} T \rho_{L}(\eta+1) \\
& \times\left[\frac{\partial^{2} \Delta \omega}{\partial \eta \partial B_{k p}} B_{l p}+\frac{\partial^{2} \Delta \omega}{\partial \eta \partial B_{l p}} B_{k p}\right], \\
C_{i j k l}^{\epsilon \alpha}=C_{i j k l}^{\alpha \zeta}= & -\delta_{i j} k_{B} T \rho_{L}(\eta+1) \frac{\partial^{2} \Delta \omega}{\partial \eta \partial M_{k l}},
\end{aligned}
$$

$$
\begin{aligned}
C_{i j k l}^{\zeta \zeta}= & \frac{1}{4} k_{B} T \rho_{L}\left[\frac{\partial^{2} \Delta \omega}{\partial B_{i p} \partial B_{k q}} B_{j p} B_{l q}+\frac{\partial^{2} \Delta \omega}{\partial B_{i p} \partial B_{k q}} B_{j p} B_{l q}\right. \\
& \left.+\frac{\partial^{2} \Delta \omega}{\partial B_{i p} \partial B_{l p}} B_{j p} B_{k q}+\frac{\partial^{2} \Delta \omega}{\partial B_{j p} \partial B_{l q}} B_{i p} B_{k q}\right], \\
C_{i j k l}^{\zeta \alpha}= & C_{i j k l}^{\alpha \zeta}=\frac{1}{2} k_{B} T \rho_{L}\left[\frac{\partial^{2} \Delta \omega}{\partial B_{i p} \partial M_{k l}} B_{j p}+\frac{\partial^{2} \Delta \omega}{\partial B_{j p} \partial M_{k l}} B_{i p}\right], \\
C_{i j k l}^{\alpha \alpha}= & k_{B} T \rho_{L} \frac{\partial^{2} \Delta \omega}{\partial M_{i j} \partial M_{k l}} .
\end{aligned}
$$

The result of the minimization of Eq. (16) is the elastic modulus tensor

$$
\mathbf{C}=\mathbf{C}^{\epsilon \epsilon}-\mathbf{C}^{\epsilon \alpha}:\left(\mathbf{C}^{\alpha \alpha}\right)^{-1}: \mathbf{C}^{\alpha \epsilon} .
$$

Since the elastic moduli tensor can be symmetrized for the fcc lattice, there are only three independent elastic moduli $C_{11}, C_{12}, C_{44}$ (in the standard Voigt notation). In the Voigt notation the coefficients are transformed according to the rules $11 \rightarrow 1,22 \rightarrow 2,23=32 \rightarrow 4$, etc., so $C_{1122} \rightarrow C_{12}$, and so on. ${ }^{32}$

In our actual calculations we rely on dimensionless quantities, such as the dimensionless segment density $\tilde{\rho}$ $\equiv \rho \sigma^{3}$, the dimensionless real lattice constants $\tilde{a} \equiv a / \sigma$, the dimensionless reciprocal lattice constants $\tilde{b} \equiv b / \sigma$, and the dimensionless root-mean-square variation of the bond length $\widetilde{\xi}=\xi / \sigma$, where $\sigma$ denotes as before the effective diameter of the beads. The dimensionless moduli $\widetilde{C}_{i j k l}$ are defined as

$$
\widetilde{C}_{i j k l}=\frac{1}{k_{B} T \tilde{\rho}_{L}} C_{i j k l} \sigma^{3}
$$

In Sec. IV we discuss the details of our calculations.

\section{CALCULATION METHOD}

Performing the differentiations of Eqs. (20)-(24), and using Eq. (10), we obtain the following expression for the elastic moduli of our model polymeric solid:

$$
\begin{aligned}
\widetilde{C}_{i j k l}^{\epsilon \epsilon}= & \delta_{i j} \delta_{k l}\left\{(\eta+1)-(\eta+1)^{2} \rho_{L} \hat{C}_{L}^{(2)}(0)\right. \\
& -\rho_{L} \sum_{\{\mathbf{q}\}} \hat{C}_{L}^{(2)}(|\mathbf{q}|) \zeta^{2}(\mathbf{q})-\eta(\eta+1)^{2} \rho_{L}^{2} \hat{C}_{L}^{(3)}(0,0) \\
& \left.-\frac{2}{(\eta+1)^{2}} \sum_{\{\mathbf{q}\}} \hat{g}(\mathbf{q}) \zeta^{2}(\mathbf{q})\right\} \\
& -\frac{1}{2} \rho_{L} \sum_{\{\mathbf{q}\}}\left[\frac{\hat{C}_{L}^{\prime \prime}(2)(|\mathbf{q}|)}{|\mathbf{q}|^{2}}-\frac{\hat{C}_{L}^{\prime(2)}(|\mathbf{q}|)}{|\mathbf{q}|^{3}}\right] \zeta^{2}(\mathbf{q}) q_{i} q_{j} q_{k} q_{l} \\
& -\sum_{\{\mathbf{q}\}}\left[\frac{\hat{g}^{\prime \prime}(\mathbf{q})}{|\mathbf{q}|^{2}}-\frac{\hat{g}^{\prime}(\mathbf{q})}{|\mathbf{q}|^{3}}\right] \zeta^{2}(\mathbf{q}) q_{i} q_{j} q_{k} q_{l},
\end{aligned}
$$




$$
\begin{aligned}
\widetilde{C}_{i j k l}^{\epsilon \alpha}= & -\frac{(\eta+1)}{2 \alpha} \delta_{i j} \delta_{k l} \\
& -\frac{1}{4} a^{2} \rho_{L} \sum_{\{\mathbf{q}\}} \frac{\hat{C}_{L}^{\prime(2)}(|\mathbf{q}|)}{|\mathbf{q}|} \zeta^{2}(\mathbf{q}) q_{i} q_{j} q_{k} q_{l} \\
& -\frac{a^{2}}{2(\eta+1)} \sum_{\{\mathbf{q}\}} \frac{\hat{g}^{\prime}(\mathbf{q})}{|\mathbf{q}|} \zeta^{2}(\mathbf{q}) q_{i} q_{j} q_{k} q_{l}, \\
\widetilde{C}_{i j k l}^{\alpha \alpha}= & \frac{(\eta+1)}{4 \alpha^{2}}\left(\delta_{i k} \delta_{j l}+\delta_{i l} \delta_{j k}\right) \\
& -\frac{1}{8} a^{4} \rho_{L} \sum_{\{\mathbf{q}\}} \hat{C}_{L}^{(2)}(|\mathbf{q}|) \zeta^{2}(\mathbf{q}) q_{i} q_{j} q_{k} q_{l} \\
& -\frac{a^{4}}{4(\eta+1)^{2}} \sum_{\{\mathbf{q}\}} \hat{g}(\mathbf{q}) \zeta^{2}(\mathbf{q}) q_{i} q_{j} q_{k} q_{l} .
\end{aligned}
$$

Here, the primed symbols indicate the derivatives with respect to $|\mathbf{q}|$. The contribution from the chain connectivity enters these expressions directly in terms that contain the Fourier transform of the step operator $\hat{g}$, and indirectly via the contribution of the direct correlation function $\hat{C}_{L}^{(2)}$. Note that in the polymer density functional theory proposed by McCoy et al., ${ }^{10}$ connectivity would be included only via the direct correlation function of the melt obtained by PRISM. It turns out, however, that for our model, which lacks any longrange bond order, the contribution from the terms containing the step operator $\hat{g}$ are relatively small, representing 2\%-3\% of the actual values of the elastic moduli for all models. One would expect these to become more important if long-range bond ordering does takes place.

To calculate the elastic moduli from Eqs. (27) to (29), we use the results of minimization of Eq. (10) for $\alpha$, $\eta$, and $\rho_{L}$, and those of the second minimization for $\Delta \alpha$, with the new density profile width $\Delta \alpha+\alpha$. We perform the summations over the reciprocal space in Eqs. (27)-(29) numerically. In passing, we note that the body-centered cubic lattice is the reciprocal lattice to the fcc lattice. It is straightforward to calculate the reciprocal vectors of fcc lattice for the whole space using its general definition. Using the results of Eqs. (27) -(29), and with help of Eq. (25), we calculate the elastic moduli $\widetilde{C}_{11}, \widetilde{C}_{12}, \widetilde{C}_{44}$ for the fcc lattice.

To show that the calculation method we employ should produce quite reasonable results, we first apply it to the hardsphere problem. Equations (16)-(29) can be applied to hard spheres or monomers by setting $\hat{g}=\hat{g}^{\prime}=\hat{g}^{\prime \prime}=0$ and using for $C_{L}^{(2)}$ the known results from the PY closure. ${ }^{21}$ It is useful to recall in this context that Jaric and Mohanty, ${ }^{21}$ and Jones, ${ }^{22}$ using the same type of DFT we use, obtained negative and therefore physically incorrect results for the so-called Poisson ratio (defined in the following). In reply to strong criticism of Frenkel and Ladd, ${ }^{23}$ provoked not only by the negative Poisson ratio, but also by a poor agreement of the theoretical moduli with results from the computer simulations, Jaric and Mohanty ${ }^{26}$ suggested that inclusion of the three-body direct correlation function Eq. (11) should in-

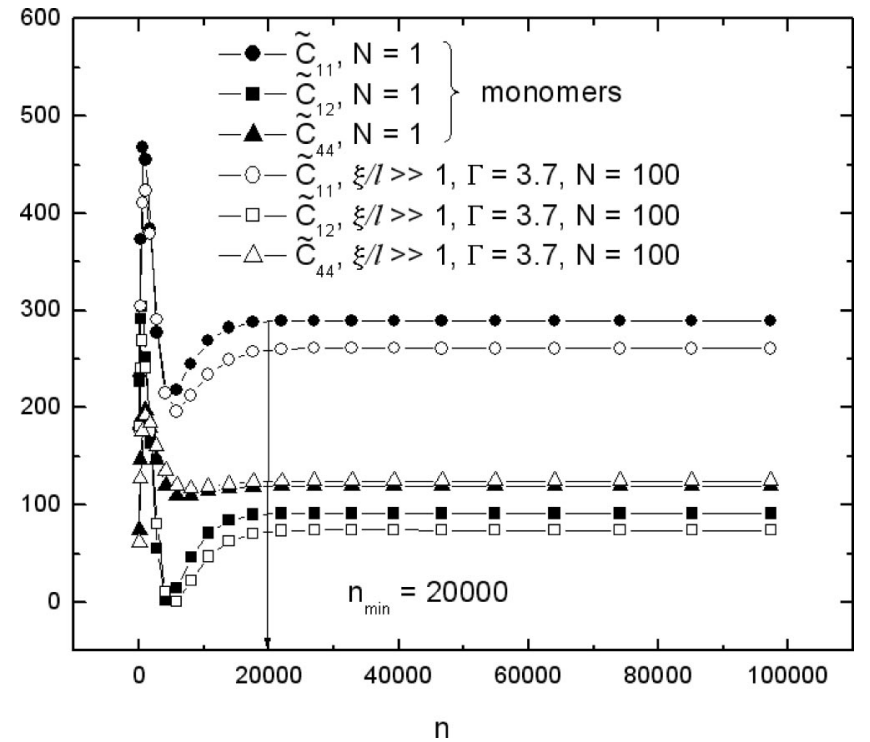

FIG. 2. Dependence of the dimensionless elastic moduli $\widetilde{C}_{11}, \widetilde{C}_{12}, \widetilde{C}_{44}$ on the number of reciprocal lattice vectors $n$ used in the summation in Eqs. (27)-(29) for the hard-sphere crystal at $\tilde{\rho}_{S}=1.12$ (closed symbols), and for a polymeric crystal with $\Gamma=3.7, \tilde{\rho}_{S}=1.14$, and $N=100$ (open symbols). The values of the moduli level off for $n>20000$.

crease the accuracy of the elastic moduli. This indeed is the reason why we have included this term in our excess free energy Eq. (10).

Another important aspect of the calculation of the elastic moduli is the number of reciprocal vectors included in the sums of Eqs. (27)-(29), which in the calculation of Jaric and Mohanty may have been not sufficiently large. ${ }^{24}$ Beforehand it is unclear how many of those need to be included in the calculations to obtain accurate values of the elastic moduli. To test this, we present in Fig. 2 the (dimensionless) elastic moduli of the solid phase as a function of the number of reciprocal lattice vectors included in the sums of Eqs. (27)(29). Results are shown for hard monomers at a density of $\tilde{\rho}_{S}=1.12$, and for model polymers (with $\xi / l \gg 1, N=100, \Gamma$ $=3.7$ ) at a density of $\tilde{\rho}_{S}=1.14$, which in fact are the densities below which the solid melts. From Fig. 2 we conclude that the values of the moduli only level off when the number of reciprocal vectors included in the calculations exceeds approximately 20000 for both monomers and polymers. This value we use in the calculations discussed next. [We need to sum over only 5832 vectors to get saturated values of $\alpha, \eta$, and $\rho_{L}$ in our minimization of Eq. (10).]

As regards the relaxing of the crystal under strain, we observe that although typical values of $\Delta \alpha$ are two to three orders of magnitude smaller than $\alpha$, the impact on the values of the elastic moduli is quite significant. Indeed, the moduli are typically smaller by a factor 4 for $\widetilde{C}_{11}$ and by a factor of 7 for $\widetilde{C}_{12}$ after the relaxation (i.e., the second minimization as explained in Sec. III), but not the shear modulus $\widetilde{C}_{44}$, which remains the same within the precision of the calculation. In all our figures we show the values of elastic moduli after the relaxation.

In Fig. 3 we compare our results for the hard-sphere fcc 


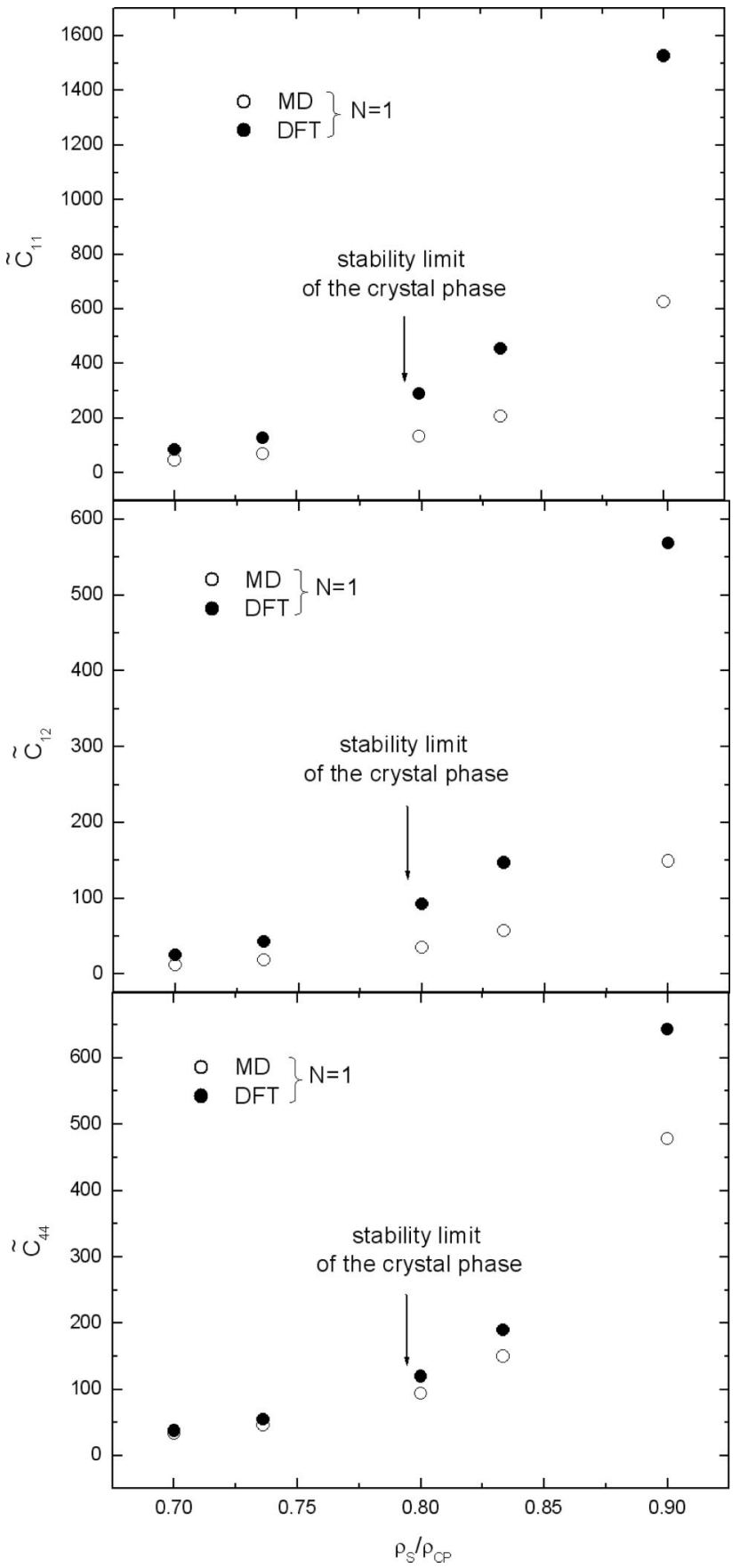

FIG. 3. DFT results for the elastic moduli of the hard-sphere crystal as a function of reduced hard-sphere crystal density $\rho_{S} / \rho_{\mathrm{CP}}$, where $\tilde{\rho}_{\mathrm{CP}}$ $=1.422$ is the (dimensionless) density of the close-packed crystal. Also indicated are the results of MD simulations of Frenkel and Ladd (Ref. 23) and our corrected DFT results for the elastic moduli (see the main text).

crystal with values obtained from molecular dynamics (MD) simulations. ${ }^{23}$ Results of both methods indicate increasing moduli with increasing density, connected with the concomitant decreasing of the free volume available to the monomeric units. The agreement between MD and DFT is quite satisfactory, albeit far from perfect. This is especially true for $\widetilde{C}_{44}$, which is the only directly accessible experimentally modulus. Whilst for all moduli the agreement between DFT and MD is fairly close to the freezing density, it deteriorates with increasing density. This, of course, is to be expected, and is connected with the expansion around the liquid reference state. Also shown are the results corrected for the assumption that the macroscopic and microscopic strains are equal, which is exact only at zero initial stress. Corrections were made following Wallace. ${ }^{31}$ For cubic crystals the solid pressure $P_{S}$ needs to be added to the moduli $C_{11}$ and $C_{44}$ in order to correct them, whilst $C_{12}$ remains unchanged. We estimated the pressure $P_{S}$ from the relation $P_{S}=P_{L}+\Delta P$, where we use the Carnahan-Starling equation of state to obtain the pressure of liquid state $P_{L}$ and the results of minimization of Eq. (7) for $\Delta P$. The corrections do not improve our results, but rather make the comparison slightly worse, especially for the low-density regime below the crystallization point.

Contrary to Jarić and Mohanty, ${ }^{21}$ we do obtain a positive Poisson ratio $\nu \equiv\left(C_{12}+P_{S}\right) /\left(C_{11}+C_{12}\right)=\left(\widetilde{C}_{12}+P_{S} \sigma^{3} /\right.$ $\left.k_{B} T\right) /\left(\widetilde{C}_{11}+\widetilde{C}_{12}\right)$. [The contribution of the pressure $P_{S}$ to the Poisson ratio, estimated from the Carnahan-Starling equation of state and the results of minimization of Eq. (7), turns out rather small, of order $10 \%$, even if we use corrected values for the elastic moduli.] A comparison of our DFT results for the Poisson ratio, and those of Frenkel's MD simulations is shown in Fig. 4. Except for the highest density tested, our values are somewhat lower than those obtained by computer simulation. ${ }^{23}$ We note that for most true solids, $\nu$ has a value between $1 / 4$ and $1 / 3$, implying a decrease in volume under tension. Our values of Poisson ratio are in this range.

In Fig. 4 we have also given a comparison of the DFT and $\mathrm{MD}$ results for the bulk modulus $B \equiv\left(C_{11}+2 C_{12}\right.$ $\left.+P_{S}\right) / 3$ and the Young's modulus $E \equiv 9 B C_{44} /\left(3 B+C_{44}\right)$, or rather their dimensionless counterparts $\widetilde{B}=B \sigma^{3} / k_{B} T \tilde{\rho}_{L}$ and $\widetilde{E}=E \sigma^{3} / k_{B} T \tilde{\rho}_{L}$. Again we observe good agreement at low densities, and less good agreement at higher densities.

A simple estimate of the Young's modulus may be obtained directly from the properties of the crystal phase, using its definition as the ratio of tensile stress and strain. In our DFT we assume the density distribution to be Gaussian with width $\alpha$. Hence, the effective potential each bead experiences around each site is harmonic, implying a force constant equal to $2 k_{B} T / \alpha a^{2}$. We thus find that the Young's modulus must be proportional to $1 / \alpha a^{3}$, or in terms of solid density $\rho_{S}$, and the Lindemann ratio $L=\sqrt{\alpha}$, as $E$ $\sim k_{B} T \rho_{S} / L^{2}$. Recall that the Lindemann ratio is defined as the root-mean-square deviation of the position of a particle from its lattice site, divided by the nearest-neighbor distance. In Fig. 4 we have plotted this estimate for $E$, using values of $\alpha$ and $a$ obtained from DFT, proving it to be remarkably accurate when compared with the simulations, except again at the highest densities.

From the above we conclude that the method that we apply is reasonably accurate in the vicinity of the crystal freezing point of hard monomers, and that it should equally be suitable for the study of hard model polymers. 


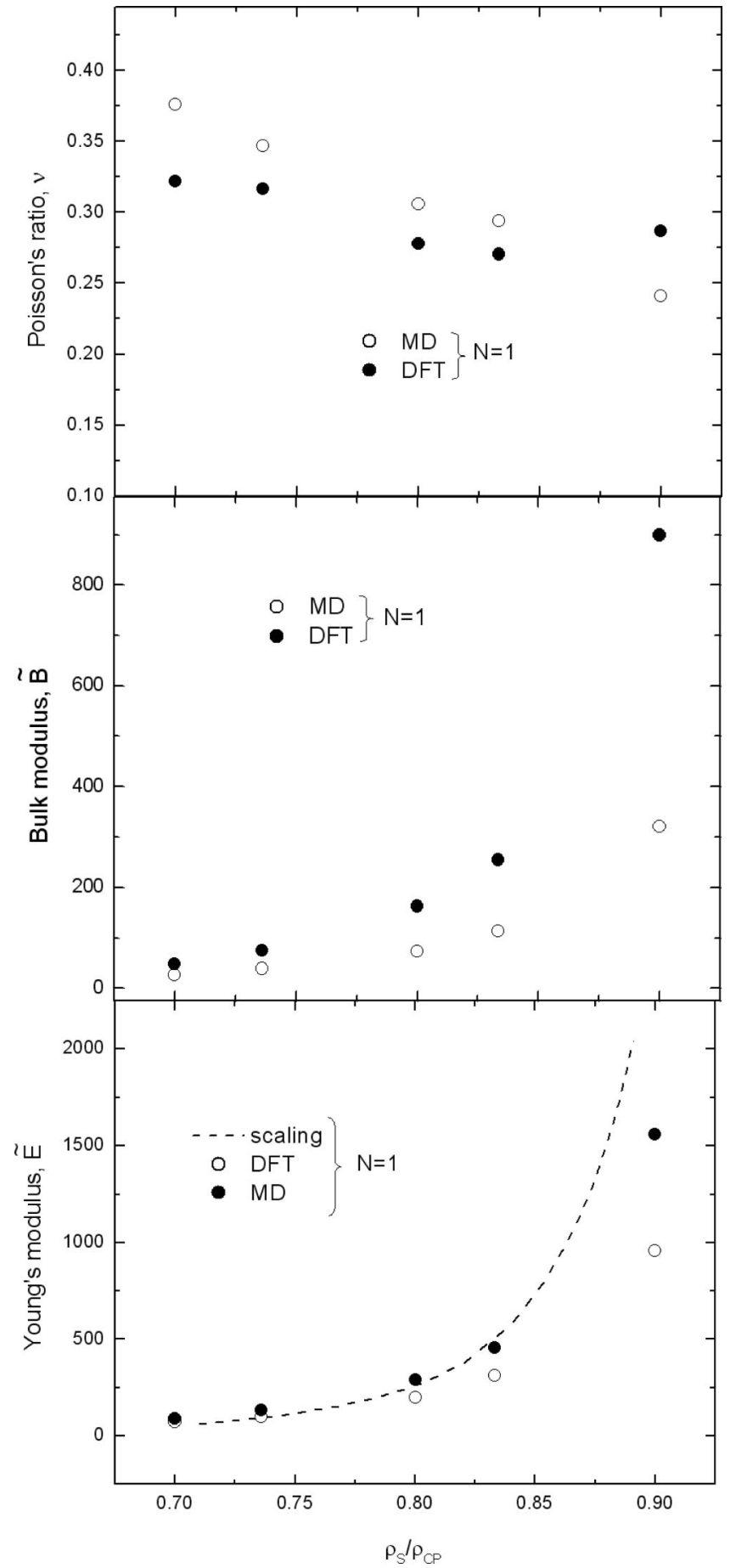

FIG. 4. The Poisson ratio $\nu$, bulk modulus $\widetilde{B}$, and Young's modulus $\widetilde{E}$ as a function of reduced hard-sphere crystal density $\rho_{S} / \rho_{\mathrm{CP}}$, where $\rho_{\mathrm{CP}}$ is the density of the close-packed crystal. The results of the DFT and of the molecular dynamics simulations of Ref. 23 are shown, as well as an estimate for the Young's modulus, discussed in the main text.

\section{RESULTS AND DISCUSSION}

We now describe the results we obtained for the model polymers, focusing on the dependence of the elastic moduli on the various system parameters. Results are presented for the cases where: (i) the fusion parameter $\Gamma$ varies at fixed density $\rho_{S}$ and degree of the polymerization $N$, (ii) the density varies at fixed fusion parameter, (iii) the degree of the polymerization of the chains varies at fixed density and fu-

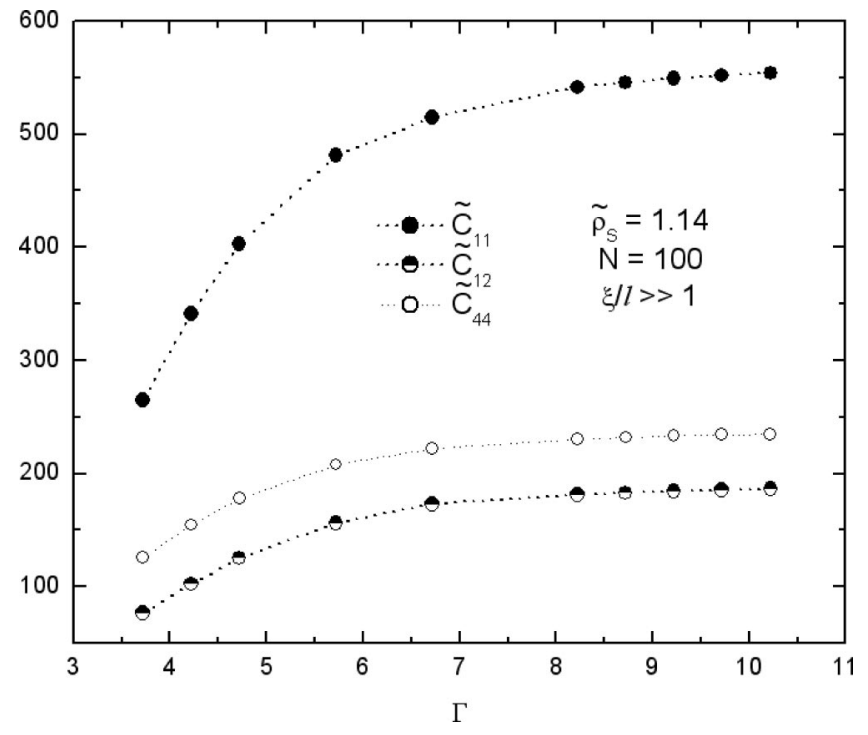

FIG. 5. Elastic moduli of the Gaussian chain as a function of the fusion parameter $\Gamma$ at fixed density $\tilde{\rho}_{S}=1.14$ and degree of polymerization $N$ $=100$.

sion parameter, and (iv) the fusion parameter and segment density vary along the freezing line at fixed degree of the polymerization. In addition, we show how the presence of bonds between the beads influences the elastic response of the crystalline phase. As we shall see, the values of the elastic constants are primarily a function of how far one is removed from the melting point of the crystal.

Figure 5 shows how the (dimensionless) elastic moduli depend on the fusion parameter $\Gamma$ for Gaussian-type chains of 100 monomeric units at a fixed density of the crystal of $\tilde{\rho}_{S}=1.14$. (Results are shown for the limit $\xi / l \gg 1$.) We find that the values of the moduli increase monotonically with $\Gamma$, leveling off in the large- $\Gamma$ regime. The reason for such a behavior is that by increasing the fusion parameter at fixed density, we move away from the equilibrium melting density, and in a way venture more deeply into the crystal phase (see also Fig. 2 of Ref. 18). For large $\Gamma$ the freezing density of the polymeric solid approaches a constant value with $\Gamma$, identical to the freezing density of hard spheres. This is caused by the dominance of packing effects in this regime. ${ }^{18}$ We check the corrections to the elastic moduli for a nonzero initial stress for a number of selected values of the fusion parameter between 3.7 and 10 under condition of phase coexistence, using the pressure calculated from the virial and compressibility routes obtained from the PRISM formalism. ${ }^{33}$ We calculated the pressure in a similar way as has been done for hard spheres to reproduce the Carnahan-Starling equation. The pressure is estimated as the sum of $2 / 3$ of pressure obtained via the compressibility route and $1 / 3$ of that obtained via the virial route. Corrected values were within $10 \%-15 \%$ of the results for zero stress. In further calculations we do not use these corrections, because of their relatively small effect and the approximate nature of our polymeric models. (Another reason is that the calculations are computationally demanding.) 


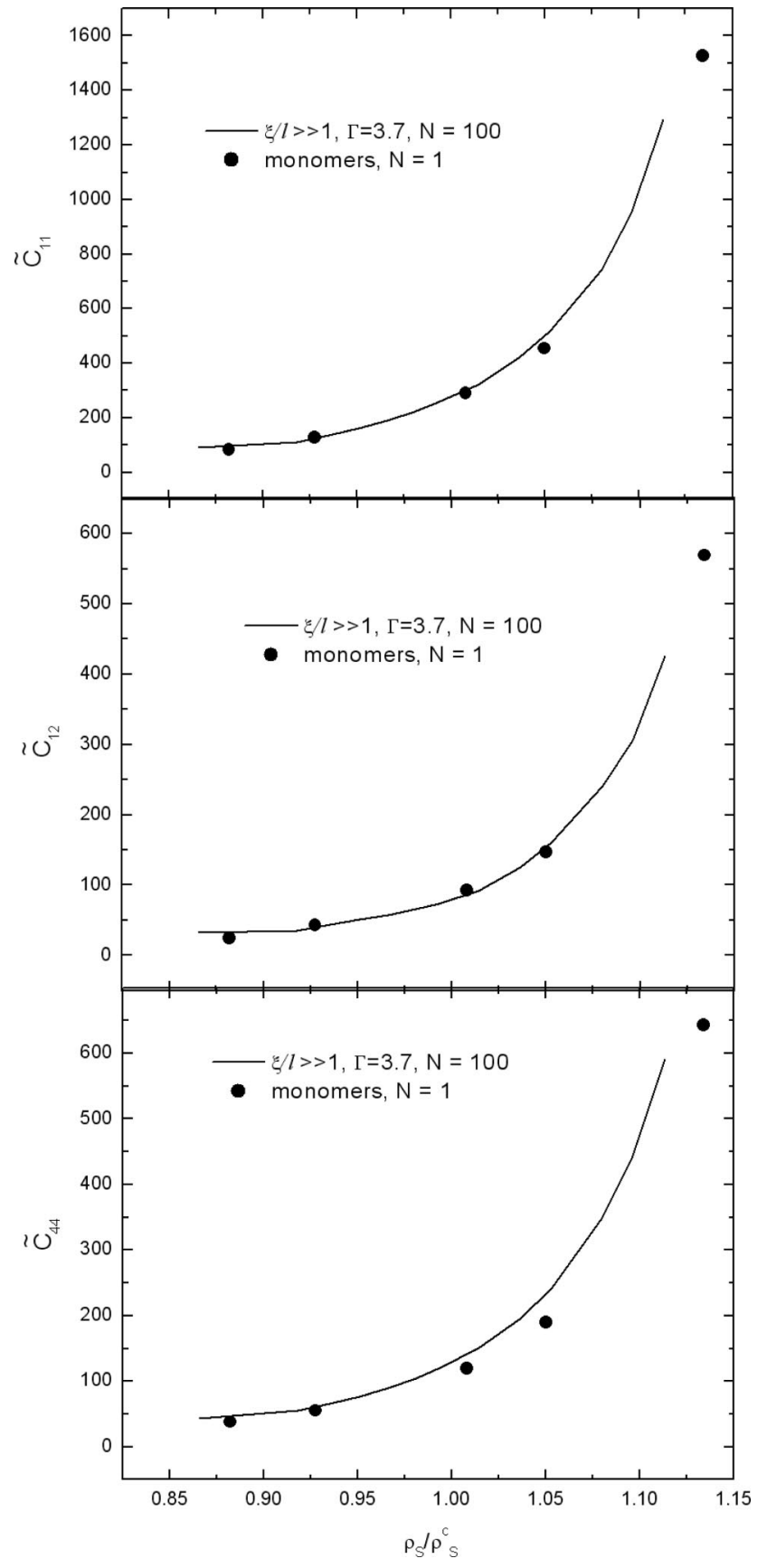

FIG. 6. Elastic moduli of the Gaussian chain limit $\xi / l \gg 1$, as a function of reduced density $\rho_{S} / \rho_{S}^{c}$, where $\rho_{S}^{c}$ is the density of the solid at coexistence with the melt, at fixed $\Gamma=3.7$ and $N=100$. Also shown are the values of the elastic moduli for the monomeric case with $N=1$.

Now we fix the fusion parameter and investigate the influence of an increase in density on the elastic moduli. In Fig. 6 we have plotted the elastic moduli again for the Gaussian limit of our model, $\xi / l \gg 1$, as a function of the ratio of the density $\rho_{S}$ of the polymeric solid and that of a crystal phase at the freezing point $\rho_{S}^{c}$ for fixed $\Gamma=3.7$ and $N=100$. For comparison also shown are our results for the hard-sphere crystal with $N=1$. For both monomers and polymers, the elastic moduli become larger with increasing density, which we attribute to a reduction of the free volume. This reduction of the free volume in turn leads to an in-

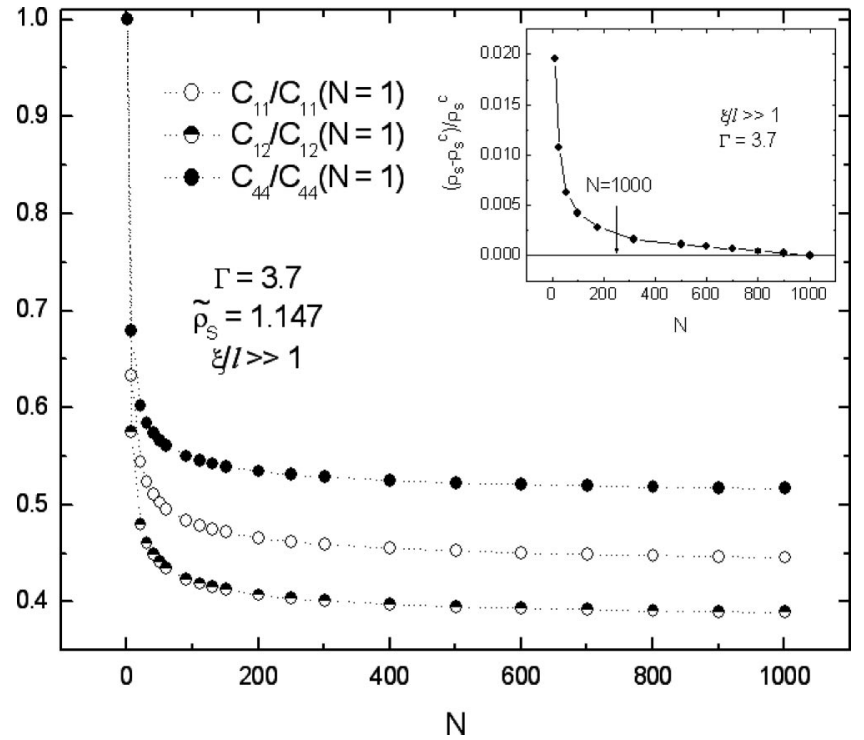

FIG. 7. Elastic moduli in the Gaussian chain limit $\xi / l \gg 1$ divided by equivalent moduli of the monomers as a function of the degree of polymerization $N$ for $\Gamma=3.7$ and $\tilde{\rho}_{S}=1.147$. The density difference between the given density and the density at the melting point are shown in the inset for the various molecular weights.

creased excluded-volume response to a deformation of the lattice.

For the metastable crystal below the coexistence point the behavior of the monomeric and the polymeric solids are almost identical, but more deeply in the crystal phase their elastic moduli diverge, albeit only modestly so. This could be due to the influence of the connectivity corrections, which are not linear in the density. It appears, at least in the Gaussian limit, that the direct influence of the connectivity on the moduli is indeed only small. This is quite a surprising conclusion, for the direct correlation functions of monomers and polymers are quite different and to leading order determine the elastic moduli. Somehow the contribution from all terms containing the information about connectivity in Eqs. (27)(29) cancel out.

In our previous work we found that shorter chains crystallize more easily than longer ones. ${ }^{18}$ Hence, by decreasing the chain length at fixed fusion parameter and density, we effectively go deeper into the crystal phase. Figure 7 confirms this trend, showing the (normalized) elastic moduli versus the molecular weight of the chains for the Gaussian limit $\xi / l \gg 1$, at fixed density $\tilde{\rho}_{S}=1.147$ and fusion parameter $\Gamma$ $=3.7$. The density was chosen such as to correspond to the freezing density of chains of length $N=1000$. For clarity, and to prove our point that the elastic response of the polymer crystal depends only on how deep we are in the crystal phase, we have also plotted, in the inset, the density difference between the given density and the density at the melting point for the various molecular weights.

Finally, we demonstrate in Fig. 8 that by increasing the stiffness of the bonds, the elastic moduli become sensitive to so-called lattice frustration effects, which also strongly influence the melting density of the crystal. ${ }^{18}$ Shown are the elastic moduli as a function of the fusion parameter. We stress 


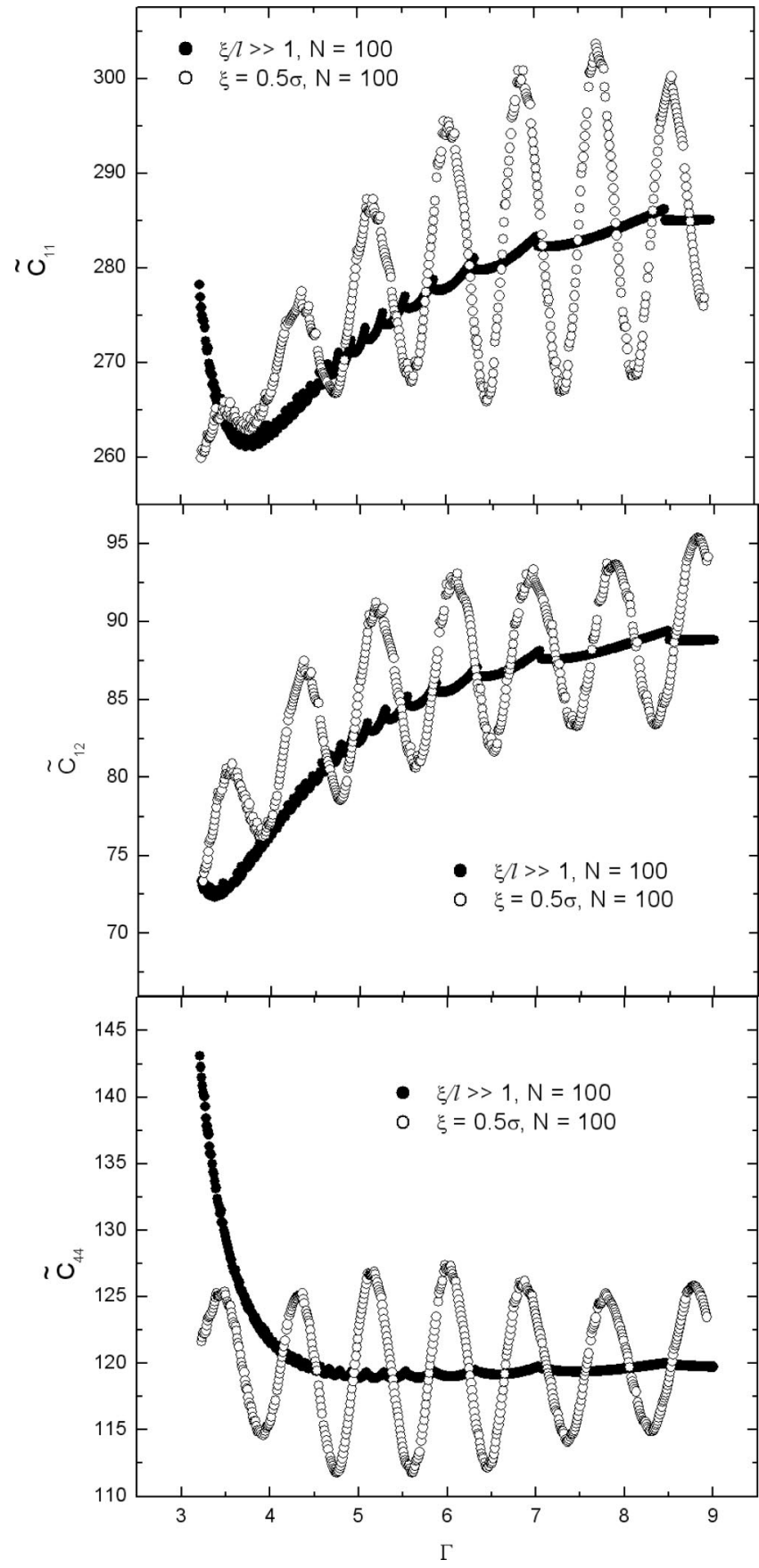

FIG. 8. Elastic moduli $\widetilde{C}_{11}, \widetilde{C}_{12}$, and $\widetilde{C}_{44}$ as a function of the fusion parameter $\Gamma$. Compared are results for the Gaussian regime $\xi / l \gg 1$ with those of the stiff-bond regime, $\widetilde{\xi}=0.5$. Note the lattice frustration effect for the model with the stiff bonds.

that, in contrast to situation of the Fig. 5, the density is now not fixed, but varies with $\Gamma$ (see Ref. 18). Compared are results for two cases, being the Gaussian case $\xi \gg l$, and the case where the chains behave more like freely hinged chains with links that extend or compress by no more than half a segment diameter, i.e., $\xi=0.5 \sigma$. An oscillatory dependence of the elastic moduli on $\Gamma$ can be observed for the stiffer bonds, which reflects a similar behavior of the freezing and melting densities, see Ref. 18. These oscillations (which become less pronounced with increasing $\Gamma$ ) are due to a mismatch between the lattice constant and the effective length of the bonds. Note that the relative variation of the elastic moduli with this parameter remains relatively small since we now remain on the melting density for all $\Gamma$ (cf. Fig. 4). This confirms once more that the direct influence of connectivity on the values of the elastic moduli is small.

That the contribution from the bonded interaction to the elastic moduli must indeed be small if bond order is absent can be demonstrated using a simple scaling estimate of the Young's modulus. Let us assume that the effective potential $U_{\text {eff }}$ associated with each bond is harmonic, so that the kernel of the step operator approximately obeys $g \sim \exp -U_{\text {eff }} /$ $k_{B} T$. (This is true close the minimum of the bondedinteraction potential.) It is then easy to show from Eq. (1) that the force constant equals $k_{B} T / \xi^{2}$. Using the same argument as in our estimate for the Young's modulus for monomers in Sec. IV, we find that the ratio between the contributions to the Young's modulus from the nonbonded interactions $\left(\sim 2 k_{B} T / \alpha a^{2}\right)$ and those from the bonded interactions $\left(\sim k_{B} T / \xi^{2} a\right)$ must be proportional to $(\Gamma / L)^{2}\left(\xi / l_{K}\right)^{2}$. For the Gaussian-chain limit $\xi / l \gg 1$ this ratio is in order of $10^{2} \Gamma^{2}$, whilst for chains that behave more like freely hinged chains, it is $\sim \Gamma^{2}$. Hence, for soft bonds the bonded interaction contributes less than a few percent to the Young's moduli, and for stiffer ones under, say, $10 \%$. This is in agreement with our more accurate DFT calculations.

Although our polymer model is crude, we nevertheless attempt to apply our predictions to actual experiment, and compare our predicted moduli with known values for polyethylene. For this purpose we need an estimate of the effective diameter of the beads in our model. The Kuhn length $l_{K}$ of polyethylene in the melt equals roughly 7 diameters $d$ of the excluded-volume sphere of each $\mathrm{CH}_{2}$ group, with $d$ $\approx 3.9 \AA .{ }^{10}$ We may therefore conclude that the volume of a single bead within our coarse-grained model must be equal to the volume of $7 \mathrm{CH}_{2}$ groups, and estimate the effective diameter of the bead for our model from the volume of a single bead as $\sigma \approx 7.46 \AA$. We fix the temperature at $T$ $=430 \mathrm{~K} .{ }^{10}$ This gives for our estimate of the fusion parameter $\Gamma \approx 4$. The solid density calculated within such an estimate is $1.0 \mathrm{~g} / \mathrm{cm}^{3}$, whilst the experimental value for the polyethylene solid is $0.996 \mathrm{~g} / \mathrm{cm}^{3}$. The dimensionless liquid density, which we need to calculate the dimension-bearing elastic moduli, is $\tilde{\rho}_{L} \approx 0.9-1.0$ for $\Gamma \approx 4$, dependent on the parameter $\xi$.

The Poisson ratio of the polymeric solid that we find for $\Gamma \approx 4$ varies with density in the range $\nu=0.22-0.26$, depending also on the precise value of the parameter $\xi$. These values are somewhat below the ones found for polyethylene in experiment, which are about $0.3-0.5 .^{34}$ The closer to the value of $1 / 2$, the lower the volume changes upon deformation. It may well be that the lack of bond ordering in our model is connected with the larger volume response upon external deformation than observed in real polymeric crystals.

The bulk modulus $B$ we find for $\Gamma \approx 4$ varies between 3.3 and $4.6 \mathrm{GPa}$ and the Young's modulus $E$ between 2.8 and $4.3 \mathrm{GPa}$, depending again on the parameter $\xi$. This has to be compared with the Young's modulus of about $2 \mathrm{GPa}$ found in experiment for isotropic polyethylene samples, ${ }^{35}$ and with 
experimental values for the bulk modulus that are of the same order of magnitude. The agreement could be coincidental, but it turns out that our results compare favorably with experimental data on typical semicrystalline polymers. ${ }^{5}$

It is quite surprising that our highly idealized polymeric model crystal can indeed describe the mechanical properties of real polymeric solids so well. A possible explanation is that in most (semicrystalline) polymeric solids the interchain interactions dominate the physics underlying their elasticity. Obviously in strongly stretched samples, which have much higher elastic moduli, especially along the chain direction, chain connectivity plays a much more prominent role. The aim of future works therefore is to incorporate long-range bond order self-consistently into the model description.

\section{CONCLUSIONS}

According to our density functional calculations, the elastic moduli of highly idealized polymeric solids are only fairly weakly dependent on the connectivity of the beadspacking effect seem to predominate. The direct influence of the polymeric corrections on the moduli is small, representing less than $5 \%$ of the numerical values of these moduli. The main influence of the connectivity is indirect via the direct correlation function of the beads that may be seen as an effective interaction potential between pairs of beads. It appears that connectivity influences the values of the elastic moduli by stabilizing or destabilizing the crystal phase of our model polymeric solid.

We found that the elastic moduli increase with increasing crystal density because of the reduction of the available free volume upon closer packing, which leads to a stronger response to external deformation. Strong oscillations of the elastic moduli as a function of fusion parameter are also found. These are most likely caused by lattice-frustration effects, discovered by us in Ref. 18. The Poisson ratio we found is positive and somewhat smaller than the one obtained experimentally, e.g., for polyethylene. Our values of the bulk and Young's moduli agree to within the same order of magnitude with the experimental values, which is an indication of the importance of the interchain interactions to the elasticity of polymeric solids.

In order to validate our polymeric DFT, we also examined the elastic moduli of hard-sphere crystals. We found that by adding the three-body direct correlation function to the expression for the grand potential, and by performing the summation over a large enough number of reciprocal-lattice vectors, the DFT significantly improved upon the original of Jaric and Mohanty. ${ }^{21}$ Indeed, we found quite reasonable agreement with the computer simulations of Frenkel and Ladd. ${ }^{23}$

\section{ACKNOWLEDGMENT}

The authors thank Joachim Wittmer (Lyon, France) for discussions and for suggesting the calculations presented in this article.

${ }^{1}$ L. R. G. Treloar, Polymer 1, 95 (1960).

${ }^{2}$ Solid Phase Processing of Polymers, edited by I. M. Ward, P. D. Coates, and M. M. Dumoulin (Hanser Gardner, Ohio, 2000).

${ }^{3}$ U. W. Gedde, Polymer Physics (Chapman \& Hall, London, 1992).

${ }^{4}$ K. Tashiro, M. Kobayashi, and H. Tadokoro, Macromolecules 10, 731 (1977).

${ }^{5}$ K. Tashiro, M. Kobayashi, and H. Tadokoro, Macromolecules 11, 908 (1978).

${ }^{6}$ K. Tashiro, M. Kobayashi, and H. Tadokoro, Macromolecules 11, 914 (1978).

${ }^{7}$ M. S. Miao, M.-L. Zhang, V. E. van Doren, C. van Alsenoy, and J. L. Martins, J. Chem. Phys. 115, 11317 (2001).

${ }^{8}$ J. C. L. Hageman, J. W. van der Horst, and R. A. de Groot, Polymer 40, 1313 (1999).

${ }^{9}$ P. J. Flory, Proc. R. Soc. London, Ser. A 234, 60 (1956).

${ }^{10}$ J. D. McCoy, K. G. Honnell, K. S. Schweizer, and J. G. Curro, J. Chem. Phys. 95, 9348 (1991).

${ }^{11}$ H. Löwen, Phys. Rep. 237, 249 (1994).

${ }^{12}$ M. Baus, J. Phys.: Condens. Matter 2, 2111 (1990).

${ }^{13}$ T. V. Ramakrishnan and M. Yussouff, Phys. Rev. B 19, 2775 (1979).

${ }^{14}$ W. A. Curtin and N. W. Ashcroft, Phys. Rev. A 32, 2909 (1985).

${ }^{15}$ M. Baus and J. L. Colot, Mol. Phys. 55, 653 (1985).

${ }^{16}$ Y. Rosenfeld, Phys. Rev. Lett. 63, 980 (1989).

${ }^{17}$ P. Tarazona, Phys. Rev. Lett. 84, 694 (2000).

${ }^{18}$ N. Sushko, P. van der Schoot, and M. A. J. Michels, J. Chem. Phys. 115, 7744 (2001).

${ }^{19}$ B. B. Laird, J. Chem. Phys. 97, 2699 (1992).

${ }^{20}$ We feel that the modified WDA method applied to our problem is unlikely to produce a better agreement. The reason is that it relies on the input of an equation of state for long polymers, obtainable, e.g., from PRISM, that is not as accurately known as that for monomers.

${ }^{21}$ M. V. Jarić and U. Mohanty, Phys. Rev. B 37, 4441 (1988).

${ }^{22}$ G. L. Jones, Mol. Phys. 61, 455 (1987).

${ }^{23}$ D. Frenkel and A. J. C. Ladd, Phys. Rev. Lett. 59, 1169 (1987).

${ }^{24}$ B. B. Laird, J. D. McCoy, and A. D. J. Haymet, J. Chem. Phys. 87, 5449 (1987).

${ }^{25}$ A. D. J. Haymet and D.W. Oxtoby, J. Chem. Phys. 74, 2559 (1981).

${ }^{26}$ M. V. Jarić and U. Mohanty, Phys. Rev. Lett. 59, 1170 (1987).

${ }^{27}$ K. S. Schweizer and J. G. Curro, Phys. Rev. Lett. 58, 246 (1987).

${ }^{28}$ P. van der Schoot, Macromolecules 33, 8497 (2000).

${ }^{29}$ Note that in the density differences in the connectivity correction term, $\rho_{S}$ should be used instead of $\rho_{L}$, however the difference turns out to be negligible in comparison with our previous calculations (Ref. 18). This is because we are interested in polymeric correction in the crystal, and solid density should be used as the reference density in this case.

${ }^{30}$ Note that our functional differs from the analogous one in Ref. 18 by factor $\eta^{2}$ because of a substitution of $\rho_{S}$ by $\rho_{L}$ in the second term in Eq. (7), which is negligibly small and does not affect the results of our calculations.

${ }^{31}$ D. C. Wallace, Solid State Phys. 25, 301 (1970).

${ }^{32}$ J. H. Weiner, Statistical Mechanics of Elasticity (Wiley, New York, 1983).

${ }^{33}$ K. S. Schweizer and J. G. Curro, J. Chem. Phys. 89, 3342 (1988).

${ }^{34}$ J. M. Powers and R. M. Caddell, Polym. Eng. Sci. 12, 432 (1972).

${ }^{35}$ Physical Properties of Polymers Handbook, edited by J. E. Mark (AIP, New York, 1996). 